\title{
The detection of COAT's disease at its early stage and management
}

\begin{abstract}
Coats disease is the potential leading cause of blindness which is an idiopathic disorder characterized by progressive deposition of intraretinal or subretinal exudates and abnormal development of retinal vasculatures known as telangiectasia that leads to exudative retinal detachment. The case shows unilateral involvement of the eye and primarily at an early age of 12 years. The cause of the disease is idiopathic and if associated with other genetic disorders emphasizes the deposition of a genetic component. The underlying histopathological problem is thought due to the abnormal permeability of capillary endothelial cells of the retina. The complications of long-standing disease may progress to total retinal detachment, leukocoria, painful Glaucoma and can be hard to differentiate from retinoblastoma. The diagnostic tools are indirect ophthalmoscopy, fluorescein angiography, CT scan, and MRI. The treatment depends on the closure of abnormal leaking vessels of the retina which is laser therapy and anti-VEGF at its early stage. The advanced stage requires surgical therapy such as scleral buckling, pars plana vitrectomy, and anterior vitrectomy for reattachment of the retina or pre retinal membrane. A careful selection therapy showed a $70 \%$ improvement in the clinical course of the disease.
\end{abstract}

Volume II Issue I - 202I

\author{
Rubina Shah \\ National eye Centre, Pakistan
}

Correspondence: Rubina Shah, National eye Centre I I Asandharoad, Lahore, Pakistan, Tel 009234240|4487, Email binashah002@gmail.com

Received:September 14, 2020 | Published: April 23, 202 |

Keywords: anti VEGF (vascular endothelial growth factor), exudative retinal detachment, and FFA fundus fluorescein angiography

\section{Case report}

The case was presented to my clinic of a 12-year-old Asian male who showed visual blurring for distance and near in the left eye for two weeks. He noticed marked blurring when closed his good eye. There weren't any other ocular symptoms. He denied pain, redness, and discharge. He denied previous spectacle history and showed a history of trauma to head with football two years back. He denied past ocular, contact lens-induced infections, surgery, or trauma. Birth history was $\mathrm{C}$-section and his maternal history was normal. The family ocular history was normal for refractive errors. He showed normal medical and social history. No known drug allergies history and he was oriented to time, place, and person.

Uncorrected distance visual acuity was 20/30OD and counting finger 10 inches in OS. Manifest spectacle correction revealed was 20/20 OD with $+0.50 /-1.25 * 05$ and no improvement with refraction in OS. Near acuity was N5 in OD and Nil in OS. Pupils were equal, round reactive with an altered glow. Extraocular muscles were unrestricted in all gazes without pain or diplopia. The cover test was orthophoric at distance and near. Goldman applanation tonometry measured 13
$\mathrm{mmHg}$ OD, 18mmHg OS at 07:25 pm. Slit-lamp biomicroscopy revealed normal adnexa, lids, lashes, puncta, and palpebral and bulbar conjunctivae, the cornea in both eyes. Siedal test was normal. Both anterior chambers appeared quiet without evidence of cell or flare, estimation of the chamber angles was 4/4 via the Von Herrick method. Both irides were flat and brown. After dilation Pupils were dilated using one drop 1\% Mydriacyl showed an assessment of fundi. The left eye showed retinal telangiectasia, vessel tortuosity, and aneurysmal dilation of capillaries in the temporal quadrant of the retina with yellow subretinal exudates. The central vision was hampered by massive exudates affecting Macula. The right eye showed normal fundus with normal macular reflex and Para papillary atrophy cup-todisc ratio of 0.30 . The diagnosed case was Coats disease stage 2(2B).

Diagnostic tools: Although, multiple diagnostic tools are required to diagnose the case such as fluorescein photography, ultrasonography to detect calcification and ancillary tests like CT scan and MRI may be helpful. We used OCT and fundus photographs with a detailed history to diagnose the case. A unilateral eye was affected with the anterior segment had normal findings (Figures 1-3).

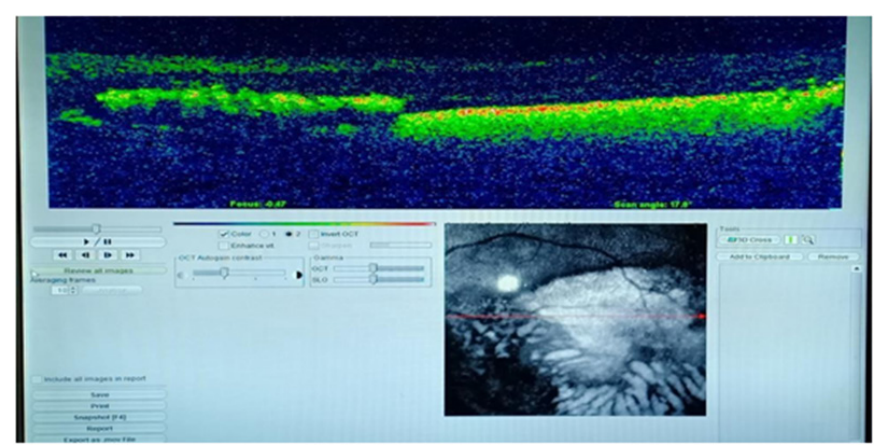

Figure I Photo courtesy national eye center.

OCT macular lines can of left eyes showed altered retinal morphology and hypereflectivity due to sub retinal exudates. However, detail visualization wasn't possible of thickness profile. 


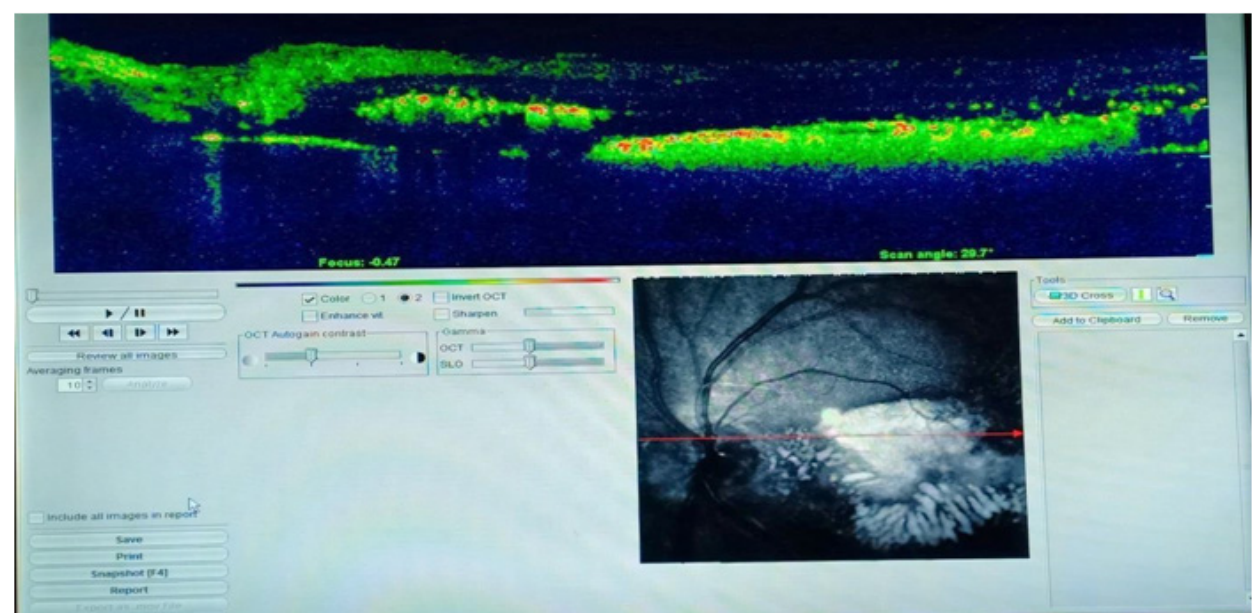

Figure $\mathbf{2}$ OCT raster scan of the same eye with a prominence of exudates.
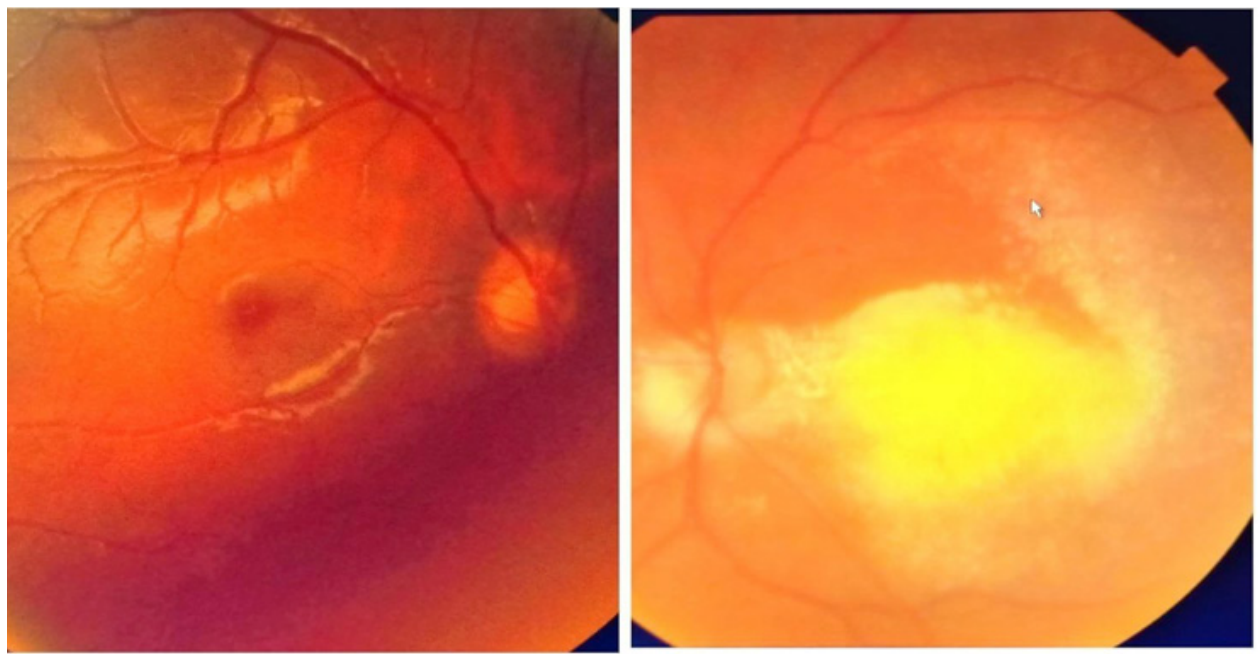

Figure 3 Photo courtesy Rubina shah national eye center.

NOTE: A Fundus photography was possible by following up on a twoweek treatment after intravitreal Patrizia (anti VEGE) Bevacizumab in the affected left eye. RIGHT fundus tortuous vessels with papillary atrophy. The LEFT eye shows regressed slight subretinal exudates with the tortuosity of vessels

\section{Differential diagnosis}

The age of presentation is a prime differential diagnosis of Coats disease. In children, all other entities must be differentiated from coats disease-causing leukocoria, intraocular mass, and strabismus.
a. Retinoblastoma
b. Persistent hyperplastic membrane (PHPM)
c. Retinopathy of prematurity ( $\mathrm{ROP}$ )
d. Toxocara granuloma
e. Familial exudative vitreoretinopathy
f. Retinal angiomatosis
g. Congenital cataract
h. Norrie disease
i. Incontinantia pigment

\section{j. Endophthalmitis and pars planitis}

In adults, it might be confused with early coats disease that includes:

I. Retinal Vein occlusion

II. Diabetic retinopathy

III. Eales disease

IV. Vasculitis

V. Juxtafoveal Telangiectasia

VI. Arterial Macroanuerysm

VII. Familial exudative retinopathy, primary vasoproliferative tumor

VIII. Epiretinal membrane with vascular leakage melanoma

\section{Choroidal capillary Hemangiomas}

Choroidal metastasis: ${ }^{1-3}$ The Challenging and principle aim of differential diagnosis consists of eliminating retinoblastoma that also shows a triad of dilated retinal vessels, retinal detachment, and subretinal mass which requires enucleation. The advances in diagnostic tools such as Radiological imaging and ultrasonography make it 
easier to differentiate between coats disease and retinoblastoma. It is therefore unlikely for enucleation that is performed as direct consequences of the diagnosis of retinoblastoma. ${ }^{4}$

\section{Disease course and classification}

The clinical course of coats disease is variable but is progressive. In quiescent stage the course is acute. A very rare case has shown regression of costs disease. ${ }^{5}$ However, the majority of the case shows massive subretinal exudation and retinal detachment. Late complication includes rubeosis iridis, secondary glaucoma, uveitis, and cataract and phthisis bulbi. The course is noticeable dismal in younger patient and virulent in the older one. The following evidence had emerged while studying a large group of samples affected by coats disease. The inferior temporal quadrant is mostly affected and with the worst visual outcome of 20/200. The risk factor of the enucleation principle was found to raise intraocular pressure and iris vascularization. ${ }^{6}$

Based on the severity of vascular abnormality, Gomez Morales classified coats disease in 1965 into five stages. $^{7}$

a. Stage 1: only focalexudates

b. Stage 2: Massive intraretinalexudates

c. Stage 3: Partial exudates with retinaldetachment

d. Stage 4: Total retinaldetachment

e. Stage 5: Complication and secondary chronic retinal detachment (Neovascular Glaucoma.

Shields et al., ${ }^{8}$ proposed a new staging classification based on large consecutive case series of coats disease that might improve in treatment and predict the future visual outcome.

That includes:

Stage 1: retinal telangiectasiaonly

Stage 2: Telangiectasia with exudates

a. Extrafovealexudation

b. Fovealexudation

Stage 3: Exudates and retinaldetachment

I. Subtotal Detachment

II. Extrafoveal

III. Foveal

Total detachment

Stage 4: Total retinal detachment and Glaucoma

Stage 5: Advance and end-stage disease

\section{Treatment and management}

The major rationale for treatment is the obliteration of retinal vessels that are affected. Laser therapy and cryotherapy are commonly used for the management of coat disease, which hampers further leakage from telangiectasia vessels and reabsorbs the exudates. A recent study has shown the intravitreal Anti-VEGF is effective for regression of exudates. Second, Laser photocoagulation was thought to be helpful for the early stages of the disease. ${ }^{9}$ Where cryotherapy is more effective for peripheral lesions and exudates..$^{10,11}$ The Techniques are useless when there is retinal detachment specifically two-quadrant of the retina. However, there is no universal given treatment to control Coats disease. In the majority, the natural course progress to Glaucoma and phthisis. However, a fewer patient has been recorded with progression. ${ }^{12}$ On the other hand, the successful treatment of retinal telangiectasia and exudates pattern was $7 \%$ and more in some patients.

The suggested classification of coat disease by Shields et al., ${ }^{8}$ can be beneficial while providing treatment and predicting the visual outcome.

1) Stage 1: The stage can be managed by frequent observation follow-ups and laser photocoagulation. The visual prognosis is favorable and the eye can be saved. However, the stage is very uncommon clinically.

2) Stage 2: (Telangiectasia and exudates) the stage can be treat by anti-Bevacizumab, laser photocoagulation, and cryotherapy which depend on the extent of the affected area or ophthalmologist preference. A good visual outcome is expected if the exudates are in one quadrant without the involvement of the fovea. In stage $2 \mathrm{~B}$ visual prognosis is good if the exudate isn't advance. But if the exudate is dense yellow nodule the prognosis worsens.

3) Stage 3A: (Subretinal detachment) cryo and photocoagulation may be helpful. Even if, detachment involves macula it can resolve when telangiectasia is eradicated. Cryotherapy is more effective than photocoagulation. Stage 3B total detachment can be managed surgically.

4) Stage 4: (Total retinal Detachment with Glaucoma) this stage may require enucleation in case of severe ocular pain.

5) Stage 5: This stage doesn't require any treatment because of a blind and comfortable eye.

Ophthalmologists preferred anti-VEGE (Patrizia) intravitreal Bevacizumab with topical Maxidex eye drop four times a day on the date of examination for stage $2 \mathrm{~B}$ Coats disease. Further, laser photocoagulation was also planned for future therapy. ${ }^{9-12}$

\section{1st Follow up: (2weeks)}

On the date of examination, there was an altered glow of pupil reflex with the torch of the left eye and subconjunctival hemorrhage. Visual acuity was counting finger 1 feet OS and 20/20 in OD. Intraocular pressure was $15 \mathrm{mmHg}$ OD and $14 \mathrm{mmHg}$ OS. The fundus examination of the left eye showed slightly regressed exudates with delineated margins. The patient was advised to undergo laser photocoagulation to leaking vessels. Topical Maxi-D was continued.

\section{2nd Follow up: (3weeks)}

On the date of examination of 3 weeks follow-up. The patient's visual acuity was hardly 20/200 OS with the normal anterior segment. Applanation tonometry was $16 \mathrm{mmHg}$ OD and $15 \mathrm{mmHg}$ OS. Fundus evaluation of affected eye showed stable lased retina and slightly improved appearance of the foveal lesion. The patient was advised with $-1.25 \mathrm{DC} * 05$ spectacles on OD and Plano in the left eye to have a comfortable distance vision. Another Patrizia intravitreal with a cryo laser was advised by an ophthalmologist.

\section{3rd Follow up}

(3weeks) on the follow-up evaluation visual acuity was 20/20 in OD and 20/200 with spectacles. Intraocular pressure was $14 \mathrm{mmHg}$ $\mathrm{OD}$ and $13 \mathrm{mmHg}$ left the eye. Fundus examination revealed a slightly 
faded macular exudate. His monocular vision tended to support very well in the better eye. The patient was educated to wear protective eyewear or sports goggles for sports and the need for regular checkups for the monitoring of eye health (Figure 4).

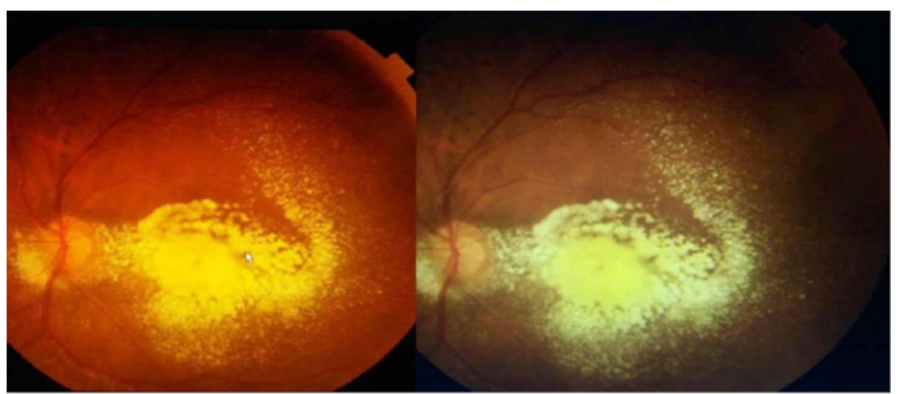

Figure 4 Fundus photography's left eye shows slightly faded macular exudates with previous laser remarks after intravitreal injection and laser photocoagulation.

\section{Discussion}

Coats disease was first described by Dr. George Coats. ${ }^{13}$ The disease was further refined by Reese ${ }^{14}$ and Shields..$^{15}$ It is categorized by aneurysmal retinal vessels with intraretinal fluid unilateral telangiectasia and, subretinal fluid (SRF), and lipid exudation. The prevalence is commonly found in young males. ${ }^{16}$ The affected eyes show thickening of outer retinal thickening and total or half exudative retinal detachment. The lipid crystal is laden due to subretinal fluid viscosity. Telangiectasia is seen with distal drop out of retinal capillary dropout and can lead to ischemic changes due to neovascularization of the disc. The disease is present in the retinal vasculature abnormality, which attains numerous aneurysms that do not often bleed. The histopathology of the retinal involved vessels shows a loss of endothelial cells and pericytes along with thickening. [16] Clinical findings depend on the altered vascular integrity of exudation. Studies have been conducted to evaluate the genetic association of Coats disease. Furthermore, several genetic such as NDP, CRB1, and PANK2 have been associated with Coats disease, but none have been proved definitively related. This suggested the chance of somatic mutation as the new lead cause. ${ }^{17}$

It is usually seen in children but some case is also noted in adults. Smithen et al., ${ }^{18}$ reported 13 patients with a mean age of 50 years in coats disease. Even though these patients have similar symptoms as that of children, the author described a slower progression in older patients that may lead to later diagnosis. Community-based research has shown that stage 3 or higher prevalence was more common at younger ages, while early stages were more common in older patients. ${ }^{19}$ The current study demonstrated that 3 out of 27 patients were over 18 years of age. Two of those patients were followed without treatment because the eye was blind.

The FFA Fundus photograph angiography can only help to visualize the telangiectatic and abnormal aneurysmal vessels which are particularly in the periphery and may be accompanied by the avascular area. ${ }^{20,21}$ The coats disease study series of adults reported areas of capillary nonperfusion that were observed of $91.7 \%$ in regions of vascular abnormalities. ${ }^{22}$ The vascular anomalies and avascular fields may appear on FFA in fellow eyes without an ophthalmoscopic finding. ${ }^{23}$ It has been shown that FFA guided laser photocoagulation results more effectively when given in the early disease stages and this may lessen the number of repeat treatments. ${ }^{24}$ The auxiliary imaging modality is FFA in terms of identifying nonperfusion areas during monitoring regression and proper treatment during follow-up.
The primary treatment involves the ablation of vascular abnormalities thus reduce intraretinal and subretinal exudation and prevent further worsening. In stage 2 or 3 of Coats disease without the involvement of severe detachment, cryotherapy and laser photocoagulation be helpful against telangiectasia and aneurysms. ${ }^{25-28}$ A certain interval is required during follow-ups for repeated laser photocoagulation or cryotherapy applications. In a study, research showed that in 17 patients from stage $2 \mathrm{~A}$ to 4 , the globe was conserved in $94 \%$, and about half of the final visual acuity had 0.4 or better 0.12 at the end of follow-up with repeated laser photocoagulation therapy.

It is difficult to apply laser photocoagulation and cryotherapy in the presence of dense exudation. The initial treatment of intravitreal triamcinolone lessens subretinal fluid and exudates, even in bullous detachment, and can, therefore, enable the application of other therapies for vascular abnormalities..$^{29,30}$ It showed fifteen patients initially treated with intravitreal $4 \mathrm{mg} / 0.1 \mathrm{~mL}$ triamcinolone injection which reduced the size of telangiectatic vessels at 1-month followup. ${ }^{29}$ Nevertheless, its complications such as cataract and Glaucoma must be kept in mind. ${ }^{31}$

The latest studies have shown that the increasing use of anti-VEGF agents can also lead to a new application for the treatment of Coats disease. $^{32-34}$ The effect has been shown the raised VEGF level in aqueous humor and increases significantly as the disease prolongs. ${ }^{35}$ The basic science behind the histopathologic review has depicted that macrophages in the subretinal space and which results in raised vascular permeability, in addition to this leads to angiogenesis due to increased expression of VEGF. ${ }^{36}$ After administering $1.25 \mathrm{mg}$ intravitreal (Patrizia) Bevacizumab before the commencement of conventional treatments such as laser coagulation and cryotherapy has been revealed influential visual outcomes. ${ }^{37}$ Villegas et al., ${ }^{38}$ applied intravitreal Bevacizumab Anti VEGF and laser photocoagulation to twenty-four advanced stage Coats' patients with stage 3 advance retinal detachment cases and observed regression of the in all cases. It has been shown that even at stage $3 \mathrm{~B}$ and 4 disease patients, an adjuvant or neoadjuvant injection of intravitreal ranibizumab may lead to partial visual recovery. ${ }^{39}$ However, the fact cannot be denied that combination therapy may lead to vitreoretinal fibrosis and tractional retinal detachment. ${ }^{40}$ In the early stage of disease laser photocoagulation and cryotherapy were commonly used modalities Intravitreal anti-VEGF and corticosteroid injections may be advantageous in lessen exudation as that of a single treatment method does not yield satisfactory results.

An advanced Coats disease vitreoretinal surgery and scleral buckling are preferred especially in (stages 3 and 4) predominantly with tractional bands or proliferative vitreoretinopathy. Additionally, cryotherapy and laser photocoagulation facilitate internal or external subretinal fluid drainage. Mutfuoglu ve Gulkilik ${ }^{33}$ did positive functional and anatomical outcomes in 5 patients undergone vitrectomy PPV, a technique of managing internal subretinal fluid drainage and silicone oil tamponade. Even though postoperative anatomic and functional achievement, functional have been seen in a small number of patients. Although, the patient in my study did not show any gross functional improvement thorough follow-ups can procrastinate the anatomic success. The advanced stage of Coats disease does not get any benefit from treatment, while some show disease progression despite undertaking treatment. It has been observed clinically that approximately one in four patients needs enucleation due to advanced changes like total retinal detachment and Neovascular glaucoma. The patient visited the national eye center none of them underwent an enucleation procedure for the painful eye. However, the vision has been affected by some patients despite treatment. 
The differential diagnosis of exudative retinal detachment should be considered in Coats' disease. Some diseases like retinoblastoma, Norrie disease, persistent hyperplastic primary vitreous, and familial exudative vitreoretinopathy are more commonly seen in children. Moreover, there is some disease that is least considered as differential diagnoses such as Eales' disease, vasoproliferative tumor, idiopathic retinal vasculitis and Neuroretinitis, and sickle-cell anemia. Mostly the referral patient visiting our hospital had an initial diagnosis of the intraocular tumor. Such patients could have well-defined using ophthalmoscopic examination and with some diagnostic methods like FFA and ultrasonography when required. Negative family history, dominantly in male and typical unilateral features, lack of vitreous opacity, abnormal vessel telangiectasia with no known mass can help in diagnosing the case. Ultrasonography and computed tomographic imaging techniques can assist to differentiate between advanced stage Coats' disease and any intraocular malignancy. The clinical course of Coats disease is a chronic disease that needs long-term follow-up evaluation. A good visual outcome can be achieved while diagnosing at the initial stage and giving a combination of therapy laser photocoagulation and anti-VEGF. Even in advance stages, the treatment might not benefit vision but the certain surgical procedure might help in saving the eye from the phthisis eye. So, the clinical practitioner must keep in mind that after regression new telangiectasia and aneurysmal changes may again encounter while following up. Hence needs proper treatment.

The disease pattern progresses gradually, so the treatment depends on the restoration of vision, and patients with an age of less the 5 have shown a prompt decline of vision while those of 10 years with the insidious pattern. Certain risk factors can aggravate vision as diffuse, post equatorial, location of exudation and telangiectasia or failure of fluid absorption and presence of retinal detachment and other ocular advance complications uveitis, rubeosis iridis, and glaucoma can aggravate visual prognosis. The visual prognosis depends vastly on the severity of the disease. It affects only one eye hence has a monocular vision thus affects stereopsis. A child adopts a natural process of if have a better eye which doesn't mean causing damage to the good eye and isn't considered as a partially sighted child. However, a monocular vision can impede depth perception and handeye coordination which may subject the child to be uncoordinated and clumsy. This may improve when the child gets older with the process of adaptation. In some cases, despite good vision in the better eye doesn't show peripheral vision. They adapt to approach or sit to make it easier to focus. It is compulsory to have a regular eye test even in the better eye by an optometrist to guard the prognosis. Only dominant eyesight must use protective measures such as eyewear or sports goggles to prevent injury. Currently, visual therapy orthoptist exercises have proven to be effective in binocular vision therapy. Several perceptual learning exercises and modern advanced technology can improve binocular vision. As for horoscopes, video games, and virtual reality receives various signals from the virtual world that the player's brain must combine to play successfully. The earliest 3D shutter glasses were introduced by the University of Nottingham to treat amblyopia using virtual reality masks and innovative video game Tetris intraocular suppression of patient dichotomic training that show marked improvement of stereo acuity.

\section{Conclusion}

Coats disease is a nonhereditary condition that is characterized by retinal telangiectasia, arterial aneurysm, exudation, and exudative retinal detachment. It is based on the founder name Coats who observed massive intraretinal exudates in 1908. It affects males than females.
Certain imaging techniques might make it easier to diagnose which includes FFA, ultrasonography, and fundus picture. The differential diagnosis must be considered especially for childhood retinoblastoma. There are various stages of disease involvement that helps to provide proper treatment in different stages. Currently, available treatment is anti-VEGF, cryotherapy, and laser photocoagulation. Although, the treatment might not directly benefit vision but prevent the eye from further advanced sequelae end-stage. The visual prognosis depends on the disease involvement of foveal from exudation and total exudative detachment which have been linked as the worst visual prognosis. The only way to manage the disease is early detection of stage 1 . The patient must be guided regarding the requirement of follow-up to manage the disease and the chances of recurrence.

\section{Acknowledgments}

None.

\section{Conflicts of interest}

There are no conflicts of interest.

\section{Funding}

None.

\section{References}

1. Deepak PE, Mahmood FM. Coats disease and persistent hyperplastic primary vitreous. Role of MRI imaging and CT radiologic. Clinics of North America. 1998;36:1119-1131.

2. Kaur B, Tylor D. Fundus hemorrhages in infancy. Surv Ophthalmol. 1992;37:1-17.

3. Scimeca G, Magargal LE, Ausburger JJ. Chronic exudative ischemic superior temporal branch retinal vein occlusion simulating Coats disease. AnnOphthalmol. 1986;18:118-120.

4. Reese AB. Telangiectasia of the retina and coats disease. Am J of Ophthalmol. 1956;42:1-8.

5. Detusch TA, Rabb MF, Jampol LM. Spontaneous regression of retinal lesion incoats disease. Acta Ophthalmol. 1982;17:169-172.

6. Shields JA, Shields CL, Honavar SG, et al. Clinical variation andcomplication of coats disease in 150 case:the 2000 sanford grifford Memorial lecture. Am J Ophthalmol. 2001;131:561-571.

7. Gomez Morale A. Coats disease: Natural history and results of treatment Am L Ophthalmol. 1965;60:885-865.

8. Shields JA, Shields CL, Honavar SG, et al. Classification and Management of Coats Disease: the 2000 procter lecture. Am J of Ophthalmol. 2001;131:572-583.

9. Haik BG. Advanced coats disease. Trans Am J Ophthalmol. 1991;89:371476.

10. Tasman W. Coats disease Clinical decisions in medical retinal disease, p204. In: TasmanW, editor. Mosby Yearbook, St Louis;1994.

11. Ridley ME, Shields JA, Brown GC, et al. Coats Disease. Evaluation and management Ophthalmology.1982;89:1381-1387.

12. Yoshizumi MO, Krieger AE, Lewis H. Viterectomy technique in late-stage coatslike exudative retinal detachment. Doc Ophthalmol. 1995;90387-394.

13. Coats G. Forms of retinal diseases with massive exudation. Graefes Arhivfür Ophthalmologie. 1912;17:440-525.

14. Grosso A, Pellegrini M, Cereda MG, et al. Pearls and pitfalls in the diagnosis and management of coats disease. Retina. 2015;35(4):614-623. 
15. Sigler EJ, Randolph JC, Calzada JI, et al. Current management of Coats disease. Surv Ophthalmol 2014;59(1):30-46.

16. Smithen LM, Brown GC, Brucker AJ, et al. Coats' disease diagnosed in adulthood. Ophthalmology. 2005;112:1072-1078.

17. Villegas VM, Gold AS, Berrocal AM, et al. Advanced Coats' disease treated with intravitreal bevacizumab combined with laser vascular ablation. Clin Ophthalmol. 2014;8:973-976.

18. Durukan AH. Coats Disease Ret Vit. 2012;20(özel sayı):115-119.

19. Blair MP, Ulrich JN, Elizabeth Hartnett M, et al. Peripheral retinal nonperfusion in fellow eyes in coats disease. Retina. 2013;33:1694-1699.

20. Suzani M, Moore AT. Intra operative fluoresce in angiography-guided treatment in children with early Coats' disease. Ophthalmology. 2015;122:1195-1202.

21. Schefler AC, Berrocal AM, Murray TG. Advanced Coats' disease. Management with repetitive aggressive laser ablation therapy. Retina. 2008;28:S38-41.

22. Küçümen RB, Görgün E, General NM, et al. Argon Laser Photocoagulation in the Current Treatment of Coats Disease. T Oft Gas. 2009;39:64-69.

23. Erol N, Topbaş S. Effectiveness of Cryotherapy and Laser Photocoagulation Treatment in Coats Disease. Ret-Vit. 2010;18:46-51.

24. Othman IS, Moussa M, Bouhaimed M. Management of lipid exudates in Coats disease by adjuvant intravitreal triamcinolone: effects and complications. Br J Ophthalmol. 2010;94:606-610.

25. Ghazi NG, Al Shamsi H, Larsson J, et al. Intravitreal triamcinolone in Coats' disease. Ophthalmology. 2012;119:648-649.

26. Sallam A, Comer RM, Chang JHShort-term safety and efficacy of intravitreal triamcinolone acetonide for uveiticmacular edema in children. Arch Ophthalmol. 2008;126:200-205.

27. Zheng XX, Jiang YR. The effect of intravitreal bevacizumab injection as the initial treatment for Coats' disease. Graefes Arch Clin Exp Ophthalmol. 2014;252:35-42.

28. Gaillard MC, Mataftsi A, Balmer A, et al. ranibizumab in the management of advanced Coats disease Stages 3B and 4:long-term outcomes. Retina. 2014;34:2275-2281.
29. Zhao Q, Peng XY, Chen FH, et al. Vascular endothelial growth factor in Coats' disease. Acta Ophthalmol. 2014;92:e225-228.

30. Zheng XX, Jiang YR. The effect of intravitreal bevacizumab injection as the initial treatment for Coats' disease. Graefes Arch Clin Exp Ophthalmol. 2014;252:35-42.

31. Ramasubramanian A, Shields CL. Bevacizumab for Coats' disease with exudativeretinal detachment and risk of vitreoretinal traction. $\mathrm{Br} J$ Ophthalmol. 2012;96:356-359.

32. Kranias G, Krebs TP. Advanced Coats' disease successfully managed withvitreoretinal surgery. Eye (Lond). 2002;16:500-501.

33. Muftuoglu G, Gulkilik G. Par's plana vitrectomy inadvanced coats'disease. Case Rep Ophthalmol. 2011;2:15-22.

34. Suesskind D, Altpeter E, Schrader M, et al. Pars plana vitrectomy for treatment of advanced Coats' disease - presentation of a modifiedsurgical technique and long-term follow-up. Graefes Arch Clin Exp Ophthalmol. 2014;252:873-879.

35. Kiratl1 H, Eldem B. Management of moderate to advanced Coats'disease. Ophthalmologica. 1998;212:19-22.

36. B Morri, B Foot, A Mulvihill,. A population-based study of Coats disease in the United Kingdom I:epidemiology and clinical features at diagnosis. Eye. 2010;24(12):1797-1801.

37. Patent WO2003092482. Ocular display apparatus for assessment and measurementof and for treatment of ocular disorders, and methods there for

38. Eastgate RM, Griffiths GD, Waddingham PE, et al. Modified virtual reality technology for treatment of amblyopia. Eye (London, England). 2006;20 (3):370-374.

39. Gareth Mitchell. Video games tackle "lazy eye”". BBCNews. 2006

40. N Herbison, S Cobb, R Gregson, I Ash, et al. Interactive binocular treatment (I-BiT; for amblyopia:results of a pilot study of 3D shutter glasses system. Eye. 2013;27(9):1077-1083. 